\title{
CHRONIC NEPHRITIS IN A NEWBORN INFANT
}

\author{
BY \\ ALBERT E. CLAIREAUX and MORTON G. PEARSON \\ From the Bernhard Baron Memorial Research Laboratories, Queen Charlotte's Hospital, \\ and the Institute of Obstetrics and Gynaecology, Hammersmith Hospital, London
}

(RECEIVED FOR PUBLICATION MARCH 3, 1955)

Renal disease other than that associated with congenital malformations is rare in the newborn infant. Formerly syphilis played an important role in the production of inflammatory renal disease in infants, but with modern methods of ante-natal care congenital syphilitic nephritis is almost unknown in this country.

Glomerular changes, consisting mainly of hyalinization and fibrosis of the glomerular tufts, were described by Herxheimer (1909) and by Schwarz (1928). These lesions were called 'congenital glomerulosclerosis by Friedman, Grayzel and Lederer (1942) who reviewed the subject in some detail. More recently Swan (1944) and Tedeschi, Halpern and Ingalls (1953) have reported similar lesions in the kidneys of infants whose mothers had suffered from rubella during pregnancy. It is felt, however, that these changes are probably vascular rather than infective in origin and are not specific manifestations of infection by rubella virus.

True glomerulonephritis is regarded as being uncommon by Zuelzer, Charles, Kurnitz, Newton and Fallon (1951) and according to Potter (1952) is unknown in the newborn infant.

Recently, however, there have been three reports of glomerulonephritis occurring in newborn infants (Thompson, 1951; Collins, 1954; Kunstadter and Rosenblum, 1954). These authors regarded the condition as an extreme rarity and were unable to find reports of similar cases in the literature. Lapage (1932) had described a case of acute haemorrhagic nephritis in the newborn, but glomerular changes were absent.

In this paper we describe a case of nephritis in a newborn infant. The renal changes were most severe and affected the glomeruli, tubules and interstitium.

\section{Case Report}

The mother was a healthy woman of 29 years of age, and this was her third pregnancy. Her first pregnancy in 1948 resulted in the birth of a female child who subsequently showed pyloric stenosis. She had a healthy female child as a result of her second pregnancy in 1951 .

During the pregnancy she seemed quite well until the beginning of the third month when she had a mild throat infection. A more severe throat infection occurred during the fifth month. Similar attacks had occurred during the previous two years. She was first seen during the seventh week of her pregnancy. Blood pressure was $11570 \mathrm{~mm} . \mathrm{Hg}$ and her urine contained no abnormal constituents. Wassermann and Kahn reactions were negative. Her haemoglobin was $13.5 \mathrm{~g}$. $\%$ and she was Group $O \mathrm{Rh}$ positive. No abnormality was seen in a radiograph of the chest. The patient thereafter attended her local county council ante-natal clinic until the 36th week when she was again seen at hospital. No abnormality was found at this examination.

Labour began spontaneously on August 9, 1954, 19 days before the estimated date of delivery. On admission to hospital a breech presentation of the foetus was diagnosed. The membranes were still intact but the foetal heart was irregular. Breech delivery was effected two hours later. The placenta weighed $794 \mathrm{~g}$. The puerperium was uneventful. On November 15, 1954, her throat was clear. A swab yielded no pathogens. The anti-streptolysin titre of the blood was 200 units $\mathrm{ml}$. Blood urea was $22 \mathrm{mg} .100 \mathrm{ml}$. and uric acid $1.7 \mathrm{mg}$. $100 \mathrm{ml}$. The patient was a male infant of $6 \mathrm{lb} .13 \mathrm{oz}$. $(3,091$ g.) birth weight. He was apnoeic at birth, had a feeble cry and took infrequent gasps. He improved after assisted breathing in a servo-respirator but indrawing of the chest persisted. Crepitations were present in both lung fields. On radiological examination, bilateral pneumothorax was found. The infant died aged 10 hours.

Necropsy. The body was that of a well developed male infant weighing $3,175 \mathrm{~g}$. No congenital abnormality was found on external examination. The tentorium was torn on both sides and there was severe bilateral subdural haemorrhage. The brain was normal.

The larynx, trachea and bronchi were healthy. There was a pneumothorax on the left side. The lungs were congested and poorly expanded. The right lung was atelectatic and sank in water. The left lung floated as a result of gross emphysema. Large emphysematous bullae were present in the interlobular fissures.

The heart and great vessels were normal. 
A few petechial haemorrhages were present in the small intestine. The liver and pancreas were healthy.

The kidneys were quite large (each weighed 26 g.). Their appearance was similar. They showed foetal lobulation and the capsule stripped easily. Their surface was generally smooth, but a few minute cysts, $0 \cdot 5-1 \mathrm{~mm}$. diameter, were present. The cysts had smooth walls and contained a little fluid. Numerous petechiae were present over the surface of the kidneys. On slicing the cortex and medulla could be easily differentiated. The cortex was congested and numerous silvery streaks were seen running at right angles to the surface. Occasional small cysts were seen. The pyramids showed a few small haemorrhages. The calyces and pelvis were healthy. The renal vessels appeared normal and the ureters and bladder were healthy.

The spleen appeared normal. The suprarenal glands, thymus, thyroid and pituitary glands were healthy.

Histology. The left lung showed numerous subpleural emphysematous bullae. Patchy intra-alveolar haemorrhage was seen in both lungs. The right lung was unexpanded. There was no pneumonia.

The liver and spleen showed no abnormality.

The capsule of the kidneys showed little change. The glomeruli were reduced to about one-third of their usual number. Of the remainder, few were completely normal. Many were atrophic and showed periglomerular concentric fibrosis (Fig. 1). A few showed proliferation of the cells of Bowman's capsule with striking

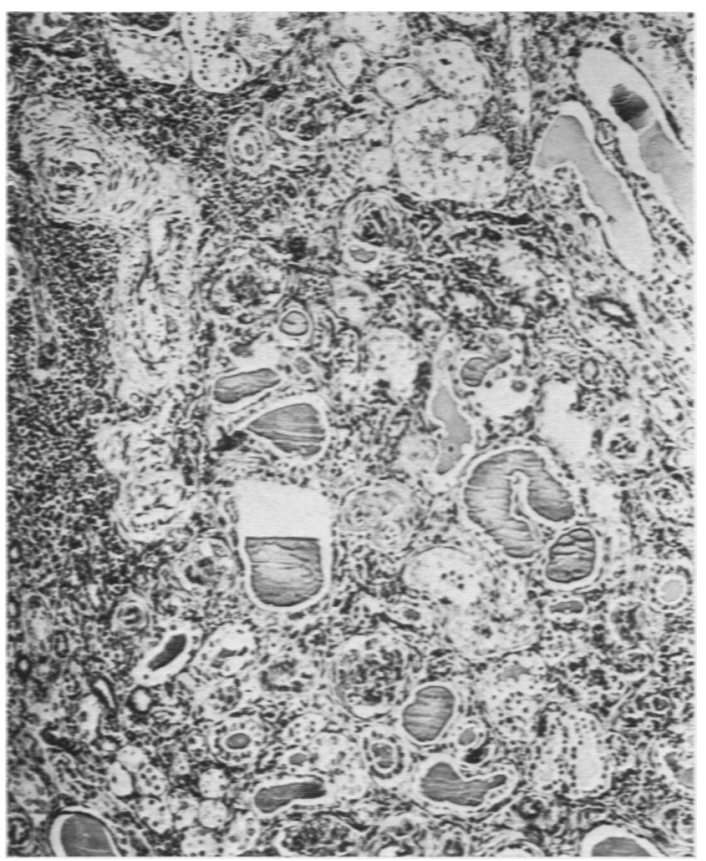

FIG. 1.-Renal cortex showing glomerular atrophy, tubular dilatation and colloid casts in the convoluted tubules. Large haemopoietic foci are present on the left. Haematoxylin and eosin $\times 75$. crescent formation and in some cases the capsular space had been obliterated by this process (Fig. 2). Pseudocrescent formation was also observed in a number of instances (Fig. 3). Here the glomeruli showed partial hyalinization and a neighbouring convoluted tubule was becoming adherent to the glomerular capsule. The latter showed epithelial proliferation and eventually the complex would have become hyalinized and surrounded by fibrous tissue. The end-result would therefore be almost indistinguishable from the crescent described above (Fig. 2). In other glomeruli capsular proliferation was less pronounced, but some adhesions were present between the glomerular tufts and the capsule. In a few instances the capsular space contained acidophile material with an occasional polymorphonuclear leucocyte. Glomeruli undergoing atrophy were present throughout the cortex, but those exhibiting proliferative change were more frequently found near the cortico-medullary junction.

The tubular changes were even more striking. The small superficial cortical cysts seen on macroscopic examination proved to be grossly dilated tubules containing acidophile fluid. About half the cortical tubules were dilated and contained colloid casts (Fig. 1). This gave the kidney a thyroid-like appearance. The dilated tubules were lined by flattened atrophic epithelium. The convoluted tubules were more affected than the collecting tubules but the latter also showed cyst formation. A few convoluted tubules showed true hypertrophy and their epithelium was thrown into papilliform

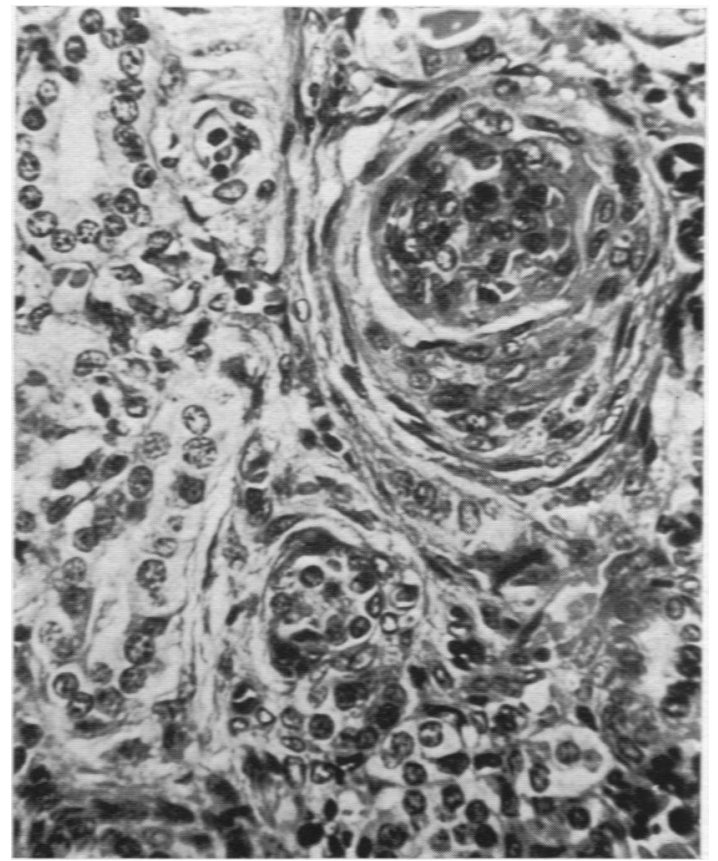

Fig. 2.-Glomerulus showing partial hyalinization of the tuft and epithelial cell proliferation of the capsule with crescent formation. Haematoxylin and eosin $\times 375$. 
folds. In addition to the acidophile colloid material a number of tubules contained polymorphonuclear leucocytes and cellular debris. Only very few tubules, however, contained purulent material and the majority contained no cells at all. A few tubules showed erosion of their walls by an inflammatory process and numerous leucocytes were present.

In addition to cystic dilatation a number of convoluted tubules showed pronounced hyaline droplet degeneration of their lining cells (Fig. 4). Their cells were pale and swollen and in places the epithelium was replaced by bright acidophile droplets. A number of convoluted tubules showed severe hyaline change and their epithelium was converted into a hyaline band (Fig. 5). In some cases two or more such hyalinized tubules had fused. No cytoplasmic inclusion bodies were seen and no organisms were found in sections stained by Gram's method.

The interstitial tissue also showed marked alteration. There were numerous large foci of extramedullary haemopoiesis throughout the cortex (Fig. 1). These were mainly normoblastic in character but there was also a considerable amount of leucopoiesis. In addition to these foci small aggregations of polymorphonuclear leucocytes and some lymphocytes were present in the peritubular connective tissue throughout the cortex. This inflammatory cell infiltration was chiefly evident in the cortex and was minimal in the medulla.

Small haemorrhages were present throughout the cortex. They were situated chiefly in the boundary zone between the cortex and the medulla. A few haemorrhages were also seen in the peripelvic connective tissue.

The interstitial connective tissue was increased in amount. This fibrosis was mainly cortical. Perivascular fibrosis was most pronounced and long strands of fibrous tissue were present in the medullary rays. These were responsible for the silvery streaking noted on macroscopic examination. The convoluted tubules were also surrounded by thickened fibrous tissue bands. The collecting tubule interstitium showed little change. There was some increase in the peripelvic connective tissue which showed slight polymorphonuclear leucocytic infiltration. The epithelium of the renal pelvis was healthy (Fig. 6). There had been some upset in renal development as a result of the inflammatory process. The outer cortex, which is the neogenic region of the organ, showed the most pronounced effect. Small groups of tubules were surrounded by concentric rings of fibrous tissue and glomeruli were absent.

The renal vessels were most noticeable for the amount of perivascular fibrosis. A number of arterioles showed hyperplastic sclerosis. Vessels in other organs were quite normal.

\section{Discussion}

In spite of the signs of glomerulitis and crescent formation in this case we believe that the fundamental lesion is a chronic, but still active, pyelonephritis. The gross dilatation of the cortical tubules which contained colloid casts, the presence of pus in a number of tubules, the increase in the interstitial connective tissue which showed infiltration with polymorphonuclear leucocytes and the glomerular atrophy with concentric pericapsular fibrosis all point to this diagnosis. We feel that only a few glomeruli showed truly proliferative lesions and these were insufficient to account for the gross alteration of the cortical architecture. The glomerular changes were probably the result of an 'alterative glomerulitis'. This change has been described in pyelonephritis by Kimmelstiel and Wilson (1936). These authors have shown that in some cases of pyelonephritis there is hyalinization of some loops of the glomerular tuft with the formation of capsular adhesions. Occasionally this is followed by capsular proliferation. They also describe a process of 'invasive glomerulitis' which probably explains the pseudo-crescent formation we observed in the kidneys of our case. In this instance there is a direct extension of the inflammatory process from the interstitial tissue, periglomerular lymphatics or tubules through the capsule into the glomerular space and tuft. This process was quite clearly demonstrated in our case (Fig. 3). A tubule containing polymorphonuclear leucocytes is seen adhering to the capsule of a glomerulus. The tubular epithelium nearest to the capsule is undergoing necrosis and will eventually become adherent. A neighbouring glomerulus in the same field shows a further stage in this process. The tubular lumen is now only a narrow cleft and the glomerulus and tubules have formed a complex mass. The capsular epithelium undergoes proliferation and eventually the whole mass is hyalinized and surrounded by concentric rings of fibrous tissue. The presence of pus in the tubules and of polymorphonuclear leucocytes in the interstitial tissue suggest that the process was still active, but these findings were not striking features of the case.

Recently Collins (1954) described a case of chronic glomerulonephritis in a newborn child who died 90 hours after birth. The illustrations indicate that the renal changes in his patient were almost exactly similar to those reported above. Collins favours a diagnosis of "chronic and subacute glomerulonephritis' instead of pyelonephritis. He excludes the latter largely on account of the severe glomerular changes in the comparative lack of inflammatory cell infiltration.

Thompson (1951) reported the occurrence of nephritis in a child who lived for 29 days after birth. The infant during this period suffered from oedema and albuminuria. Red cells, pus cells, hyaline and granular casts were present in the urine. Histological examination of the kidneys showed hyaliniza- 


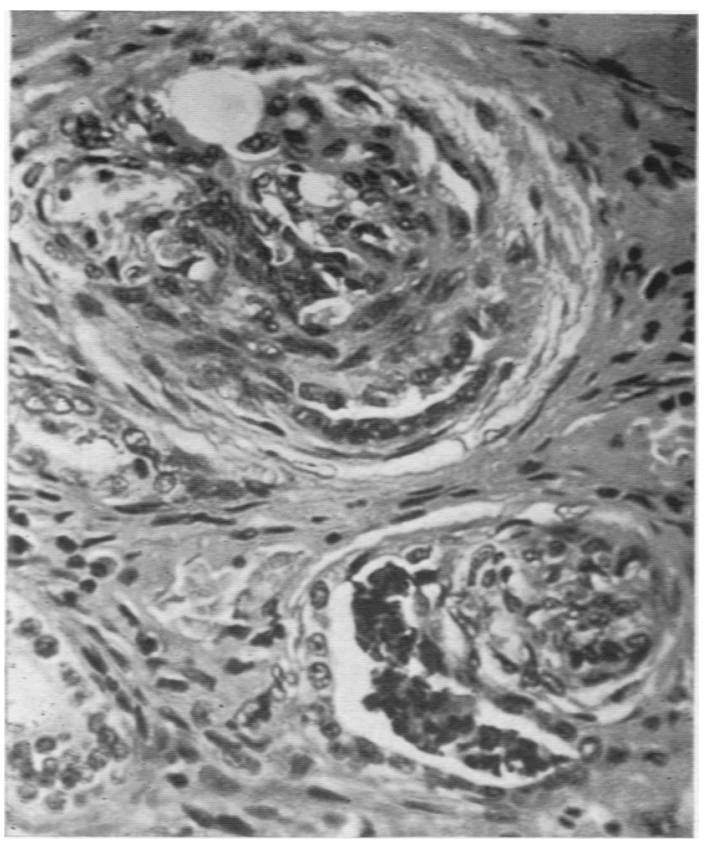

Fig. 3.-Two glomeruli with adherent tubules. On the top left the convoluted tubule is a narrow slit attached to the glomerular capsule and surrounded by fibrous tissue. On the right the tubule contains pus cells and debris. The inner wall of the tubule is becoming attached to the glomerulus. Haematoxylin and eosin $\times 375$.

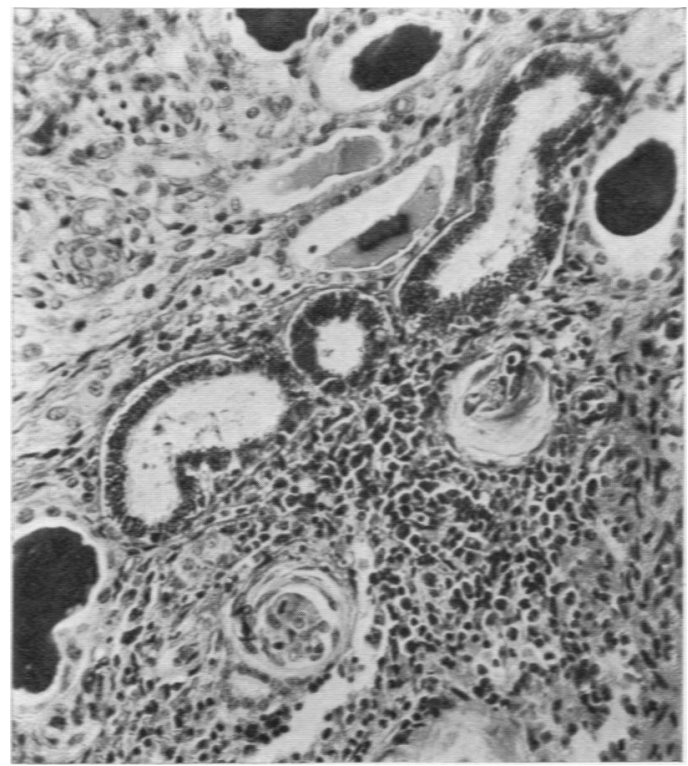

FIG. 4.-Three dilated tubules showing pronounced hyaline droplet degeneration. Picro-Mallory $\times 175$.

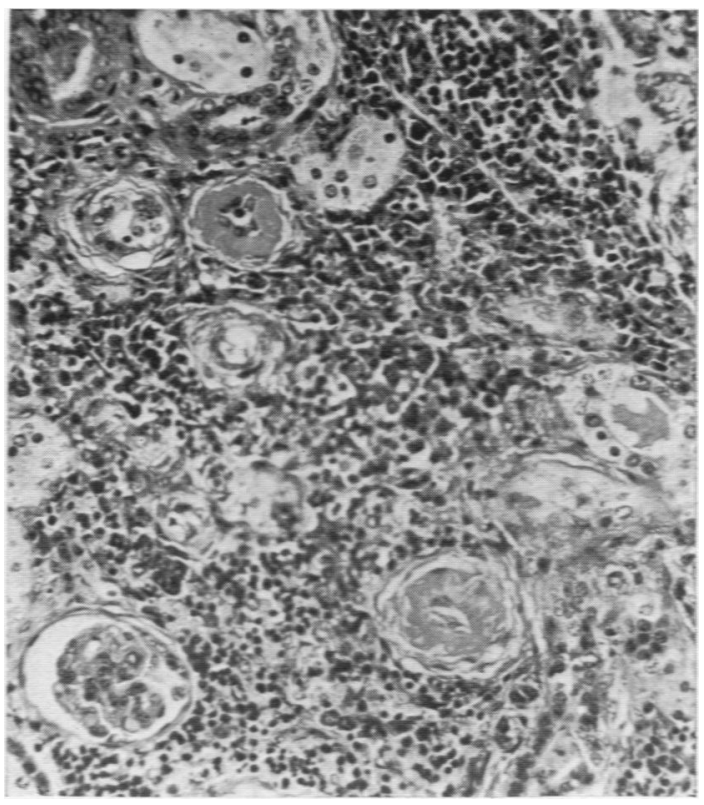

FiG. 5.-Renal cortex showing two completely hyalinized tubules with peritubular fibrosis. There is marked haemopoietic activity in the interstitium. Haematoxylin and eosin $\times 175$.

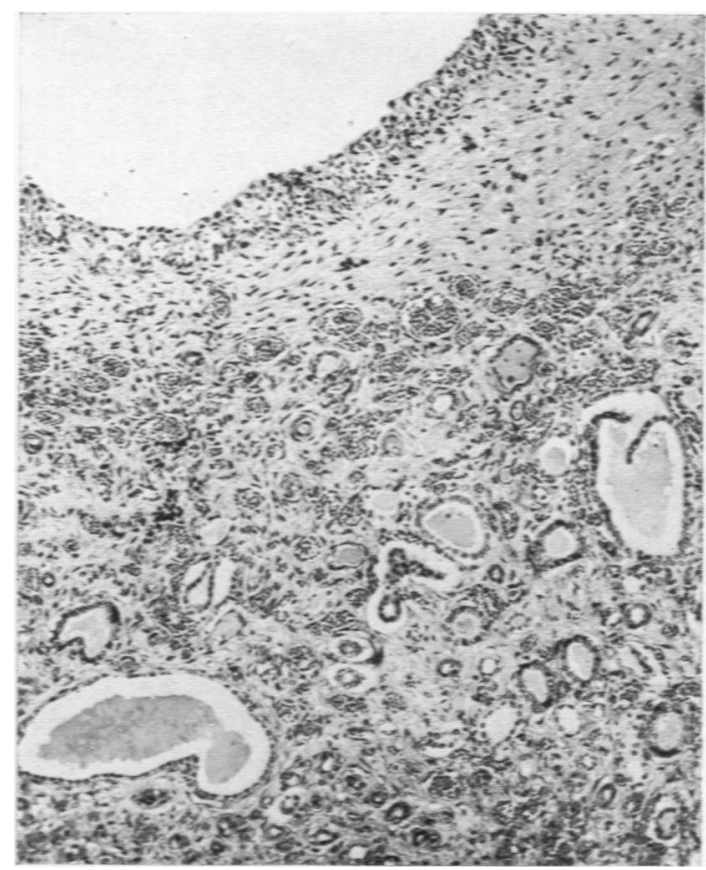

Fig. 6.-Medulla and pelvis. The stratified epithelium of the pelvis is intact. The subepithelial connective tissue shows slight infiltration with polymorphs. The medulla is congested and dilated tubules contain colloid casts. Haematoxylin and cosin $\times 75$.

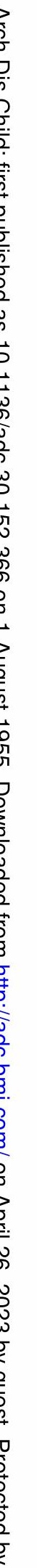


tion of some glomeruli while others showed capsular adhesions, crescent formation and increased cellularity of the tufts. The proximal convoluted tubules were lined by a partially vacuolated epithelium and the collecting tubules contained casts and leucocytes. There was marked periarterial fibrosis and the arterioles showed hyperplastic sclerosis. The periarterial fibrosis was explained on the basis of hypertensive change. The author believes that this was a case of glomerulonephritis of some standing. The considerable pyuria and the cellular infiltration of the renal stroma suggest that this view may be incorrect and that this was possibly another example of pyelonephritis.

More recently Kunstadter and Rosenblum (1954) have described a case of neonatal glomerulonephritis and the nephrotic syndrome. The infant was born prematurely and died 72 days later. During this time the infant had shown pitting oedema over the face and trunk. The infant was very anaemic and required several blood transfusions. The urine contained albumin, red cells and casts. At necropsy the kidneys showed reddening of the cortico-medullary junction and yellow streaking perpendicular to the cortical surface. On histological examination the glomeruli were small, hyperaemic and more cellular than usual. A few glomeruli showed proliferation of the cells of Bowman's capsule with some adhesions. The tubular epithelium was swollen, granular and vacuolated. The authors believe that this was an example of subacute glomerulonephritis with nephrosis.

Not one of these recent accounts shows that the diagnosis of glomerulonephritis has been established beyond any reasonable doubt, and Potter (1952) is probably still correct in her opinion that it does not occur in the newborn.

The pathogenesis of nephritis in the newborn is obscure. Collins (1954) and Thompson (1951) have suggested an allergic or hypersensitivity basis for the occurrence of glomerulonephritis in their patients.

It is interesting to note that the mother of the child reported by Thompson had suffered from a sore throat during the sixth month of her pregnancy. The mother of the infant in the present case had a throat infection twice during her pregnancy, at the third month and again during the fifth month. Whether a hypersensitivity phenomenon following on these throat infections during early pregnancy could explain the occurrence of a pyelonephritis in the present case is rather doubtful. There are certain features which favour such a supposition. The entire histological picture is rather unusual. While we are satisfield that this is not a true glomerulo- nephritis, the pronounced glomerulitis and tubular hyalinization are peculiar features. Furthermore, the intense perivascular fibrosis and pronounced periarterial cellular infiltration bear a faint resemblance to the appearance found in the healing stages of polyarteritis nodosa. The latter has been ascribed to a hypersensitivity reaction. The lack of severe inflammatory change in the renal calyces and pelvis also suggests that we are not dealing with a typical bacterial infection of the kidney.

If this pyelonephritis is to be regarded as the result of a bacterial infection we have to decide the source of infection and there seem to be only two possibilities: first, a maternal bacteraemia with transmission of the organisms to the foetal blood stream via the placenta, or secondly an infection of the liquor amnii which is then swallowed by the foetus and absorbed from the gut. The first explanation is improbable since organisms are unlikely to pass the placental barrier without causing a placental lesion and there was no evidence of this in the present case. The second supposition is more plausible, but there is no evidence that it did occur. The liquor amnii was not malodorous and no inflammatory lesions were found in the infant's lungs. There is thus no clear evidence to support the infective origin of the renal lesions and no organisms were found in the sections examined.

The changes in the renal cortex which were found in the present case are of special interest since they would seem to form the basis of the so-called 'chronic interstitial nephritis' in older children which was described by Mitchell (1930). A case of a slowly progressive nephritis associated with marked skeletal changes in a child of 5 years has already been recorded by one of us (Claireaux, 1953). The kidneys in this patient showed marked glomerular hyalinization with pericapsular fibrosis and severe interstitial fibrosis. A number of tubules contained pus. There was no congenital abnormality of the urinary tract. It is almost certain that this was an example of a chronic but still active pyelonephritis. Weiss and Parker (1939) are firmly convinced that all these cases of so-called interstitial nephritis associated with renal dwarfism are, in reality, examples of chronic pyelonephritis. Thus older infants and children who show an osteodystrophy associated with advanced renal disease have almost certainly suffered from pyelonephritis for some considerable time. Craig (1935) has shown that acute pyelitis and pyelonephritis are not uncommon in the neonatal period. The majority of patients recover but in a few instances the renal disease may become chronic with the development of hypertension and bone lesions in later life. The case 
we have described above would seem to indicate that the fundamental renal lesion can occur even in utero.

\section{Summary}

A case is reported of chronic pyelonephritis in a newborn infant. In addition to advanced tubular changes the kidneys showed unusual proliferative lesions in the glomeruli which may sometimes be mistaken for glomerulonephritis.

The pathogenesis of this condition is obscure, but is possibly the result of a hypersensitivity reaction to an infection in the mother during early pregnancy.

It is suggested that this unusual form of pyelonephritis may form the basis of the so-called chronic interstitial nephritis found in older children with renal osteodystrophy.

Claireaux A E (1953) Rerences

Claireaux, A. E. (1953). J. Path. Bact., 65, 291

Collins, R. D. (1954). Amer. J. Dis. Child., 87, 478.

Craig. W. S. (1935). Archives of Disease in Childhood, 10, 337.

Friedman, H. H., Grayzel, D. M. and Lederer, M. (1942). Amer. J. Path. 18, 699 .

Herxheimer, G. (1909). Frankfurt. Z. Path., 2, 138.

Kimmelstiel, P. and Wilson, C. (1936). Amer. J. Path., 12, 99.

Kunstadter, R. H. and Rosenblum, L. (1954). Amer. J. Dis. Child., 88,611 .

Lapage, C. P. (1932). Archives of Disease in Childhood, 7, 235.

Mitchell, A. G. (1930). Amer. J. Dis. Child., 40, 101.

Potter, E. L. (1952). Pathology of the Fetus and the Newborn, p. 361. Chicago.

Schwarz L. (1928). Virchows Arch. path. Anat., 267, 654.

Swan, C. (1944). J. Path. Bact., 56, 289.

Tedeschi, C. G., Helpern, M. M. and Ingalls, T. H. (1953). New Engl. J. Med., 249, 439.

Thompson, A. C. (1951). W. Va med. J., 47, 16

Weiss, S. and Parker, F. (1939). Medicine, 18, 221

Zuelzer, W. W., Charies, S., Kurnetz, R., Newton, W. A. and Fallon R. (1951). Amer. J. Dis. Child., 81, 1. 